Research Article

\title{
Enzyme Producing Activity of Probiotics and Preparation of Compound Enzyme
}

\author{
Ruokun Yi, ${ }^{1}$ Yanni Pan, ${ }^{1}$ Xingyao Long, ${ }^{1}$ Fang Tan $\left(\mathbb{D},{ }^{2}\right.$ and Xin Zhao $\mathbb{D}^{1}$ \\ ${ }^{1}$ Chongqing Collaborative Innovation Center for Functional Food, Chongqing Engineering Research Center of Functional Food, \\ Chongqing Engineering Laboratory for Research and Development of Functional Food, Chongqing University of Education, \\ Chongqing 400065, China \\ ${ }^{2}$ Department of Public Health, Our Lady of Fatima University, Valenzuela 838, Philippines
}

Correspondence should be addressed to Fang Tan; tanfang@foods.ac.cn and Xin Zhao; zhaoxin@cque.edu.cn

Received 15 July 2020; Revised 15 August 2020; Accepted 8 September 2020; Published 30 September 2020

Academic Editor: Tifeng Jiao

Copyright (c) 2020 Ruokun Yi et al. This is an open access article distributed under the Creative Commons Attribution License, which permits unrestricted use, distribution, and reproduction in any medium, provided the original work is properly cited.

\begin{abstract}
Probiotics are a group of active microorganisms, which benefit the host by colonizing and changing the composition of host flora. It is of great significance to promote the development of human gastrointestinal nutrition and health by regulating the host mucosal and systemic immune function or regulating the balance of intestinal flora. The purpose of this study is to analyze the production activity of the enzyme, evaluate its biological characteristics and safety as a preventive drug, and provide reference for the research of enzyme production and compound enzyme preparation by probiotics. In this study, four groups of probiotics were set up: Clostridium butyricum experimental group, Lactobacillus plantarum experimental group, drinking water control group, and Bacillus licheniformis experimental group. In addition, a variety of complex enzyme experiments were set up to study the influence on the digestive tract and single factor experiment. The results showed that probiotics and compound enzyme preparations could significantly promote the intestinal digestibility. Under the effect of probiotics, the weight of the chicken was almost 1 Jin heavier than that of the control group, and the average digestibility was increased by $4.3 \%$. The effect of the enzyme on digestibility is stronger than that of probiotics, but the final effect tends to be stable.
\end{abstract}

\section{Introduction}

1.1. Background and Significance. With the increasing serious problems of antibiotic resistance and antibiotic residues, it has become a global research topic to develop green feed additive products which can replace antibiotics. Microbial agents can supplement, adapt, and maintain the balance of intestinal microorganisms, which can be used for disease prevention and treatment, promoting health, and enhancing productivity. In recent years, the mushroom technology in China has developed rapidly, and the large-scale industrial production has gradually developed. The quantity of residual substrate mushroom bran increased rapidly after harvest. The fungus bran was rich in mycelium and rich in nutrients. The rational utilization of edible fungus bran became an urgent problem in the edible fungus industry, and the production of probiotics from mushroom bran provided a solution.
1.2. Related Work. The role of probiotics in reducing gastrointestinal inflammation and preventing colorectal cancer has been widely confirmed. However, the immunomodulatory effect of probiotics on the growth of extraintestinal tumors and its mechanism are still unclear. Here, Li used a mouse model and genome sequencing to study the efficacy of probiotic feeding in controlling liver cancer and the potential mechanism of inhibiting tumor progression. Prohep is a new probiotic mixture, which can significantly slow down the growth of tumor and reduce the tumor volume and weight by $40 \%$ compared with the control group. From the point of view of mechanism, the levels of IL-17 cytokines and their main producer Th17 cells decreased sharply, which played a key role in tumor reduction after probiotics feeding. Cell staining showed that the main reason for the decrease of 
Th17 cells in the probiotics treatment group was the lower migration frequency of Th17 cells in intestinal and peripheral blood [1].

In order to study the probiotic colonies, Tanaka established an optimized fluorescence in situ hybridization (FISH) method to detect and count the host borne probiotics in abalone intestines. The samples were fixed with $4 \%$ paraformaldehyde and hybridized with TAMRA-labeled probe (RPT probe) at $40^{\circ} \mathrm{C}$ for 3 hours. Strain Ab1 is obviously different from other control bacteria (Enterococcus, Lactobacillus). The fluorescence signal of RPT positive bacteria can be easily distinguished from any detectable background noise. RPT positive bacteria were detected in both probiotics and control abalone, but the number of RPT positive bacteria in abalone with probiotics was two orders of magnitude higher than that of the control. In addition, the average number of RPT positive bacteria in the intestinal attachment zone (abalone supplemented by probiotics) was two orders of magnitude higher than that in the free living area. A single colony from each group was identified by the biochemical method (id32c system) and molecular biological method (sequencing of D1/D2 domain of 26srdna and its 1-5.8-its2rdna region). Both methods can identify candida. The cytotoxicity, adhesion, surface properties, hemolytic activity, and survival rate of famata strain Y5 in simulated gastrointestinal environment were studied [2].

To isolate probiotics from pig intestines, Balasingham collected 63 isolates (24 caeca, 24 colonic mucosal scraps, and 15 rectal swabs) from Yorkshire pigs. The isolate was inoculated in Man Rogosa Sharpe broth at $37^{\circ} \mathrm{C}$ and $5 \% \mathrm{CO}_{2}$ for 48 hours, and morphological identification was performed. The colony with gram positive bacilli was selected for further physiological and biochemical identification test. Each selected isolate was tested twice and three times with the standard protocol. The probiotic characteristics among the identified species were determined through a number of tests related to $\mathrm{pH}$ tolerance, bile tolerance, and antimicrobial activity. Morphological identification showed that only 23 strains of gram positive bacilli were detected. Physiological tests on these 23 strains further showed that four of them did not show any growth under all the studied conditions. The remaining 19 strains were detected by biochemical methods [3].

1.3. Innovation. Using the method of single factor experiment, this paper studies the $\beta$-amylase, acid protease, and neutral protease which have good effect in industry at present, and their functions in reducing sugar content and digestibility of digestive tract, etc., and roughly determines the best kind and dosage of enzymes. Then, response surface methodology was used to optimize the selected enzymes and develop a new composite enzyme.

\section{Function of Probiotics and Enzymes}

2.1. Compound Enzyme Preparation. Compound enzyme preparation is one or more preparations of a single enzyme as the main body being mixed or fermented with other single enzyme preparation to form one or more microorganisms [4]. There are many factors that affect the digestibility of specific nutrients. They are not only related to animal species, age, and normal period but also related to food composition and processing technology. According to the digestion characteristics and feed composition of different livestock and poultry, specific enzyme preparation can be prepared for different animals and poultry. Different kinds of food may also form different specific enzymes [5]. The use of specific complex enzyme preparation can degrade a variety of food substrates (a variety of antinutrients and a variety of nutrients), and different kinds of enzymes also have synergistic effect, which can maximize the nutritional value of food [6].

At present, the enzyme feed preparations commonly used in livestock and poultry production can be divided into the following categories according to their functional characteristics: (1) compound feed enzymes mainly composed of protein and amylase, which are mainly used to supplement the deficiency of congenital enzymes. (2) Compound feed enzyme mainly composed of $\beta$-glucanase and xylanase. This enzyme preparation is mainly used in the feed with grains as the main raw material, such as barley, oats, wheat, and rye, in order to neutralize the antinutritional effect of NSP. (3) Cellulose and pectinase are the main enzymes in compound food. This enzyme is mainly produced by trichoderma, aspergillus, and penicillium. Its main function is to destroy the plant cell wall, release nutrients to the cells, easily contact with digestive enzymes, eliminate antinutritional ingredients in the diet, and reduce the viscosity of gastrointestinal contents and promote digestion and absorption of animals. (4) Feed enzyme is composed of cellulose, protease, amylase, glucoamylase, glucanase, and pectinase. This compound enzyme preparation combines the common characteristics of these enzyme systems and has good nutritional effect $[7,8]$.

2.2. Probiotics. From the beginning of human civilization, fermented milk may have become the first food containing active microorganisms. The concept of probiotics comes from Latin, meaning life. It is produced by organic substances, and the substances can stimulate the development of another substance [9]. Later, after continuous exploration by experts and researchers, it was defined as a kind of sustainable substance, which can be properly added, harmless to animals, and can ensure the health of animals [10]. As probiotics, they must meet the following conditions: (1) they must be able to survive in the intestinal transport. (2) They must be able to adhere to the animal surface, the mucous membrane and its colonization in the gut. (3) In the process of survival, they must resist the external environment. (4) They must be safe and harmless to animals. (5) It turns out to be good for animals. (6) Taxonomic identification was carried out in genera, species, and strains. Conclusions of the current requirements are Bifidobacterium, Bacillus, and yeast $[11,12]$.

2.3. Research Methods of Enzyme Producing Activity of Probiotics. At present, researchers have carried out a large number of in vitro and in vivo experiments to confirm that 
probiotics have a high antioxidant effect [13]. The antioxidant effects of probiotics are as follows: reactive oxygen species and free root scanning, metal chelating ions, oxygen reduction control system in preventive agents, activation of antioxidant system, reduction of metabolic capacity after absorption by the body, and lipid lowering effect [14]. At present, the research on antioxidant activity of disease prevention drugs mainly focuses on the scanning ability of free roots and the detection of antioxidant enzyme activity. Among them, the most extensive research involves the activity of antioxidant enzymes derived from probiotics. For example, peroxide disulfide (SOD) can eliminate free roots in the body, while aniline peroxide can produce water [15]. Catalase (CAT) is a porphyrin-binding enzyme containing iron. Its main function is to participate in the active metabolism of oxygen and scavenge hydrogen peroxide produced in the process of metabolism. The main enzyme systems in vivo are SOD, CAT, and glutathione peroxidase $[16,17]$.

In addition to producing antioxidant-related enzymes, probiotics also have other enzyme-related functions. Probiotics can reduce cholesterol, which is directly related to the production of bile salt hydrolase. Bile salt hydrolase can combine with the cholate solution to reduce serum cholesterol [18]. A strain of Lactobacillus casei can produce cholesterol hydrolase, and its cholesterol degrading ability can reach $35.74 \%$. Lactobacillus plantarum contains bile salt lyase gene and produces bile salt hydrolase. In addition, studies have shown that some prebiotics, such as Lactobacillus reuteri and Bacillus, can produce amylase $[19,20]$. In addition, prophylactic drugs may also produce enzymes related to lactose reduction, among which $\alpha$-lactosidase can hydrolyze nonreducing lactose glycosidase compounds related to bonds, and $\beta$-lactosidase can catalyze the decomposition of lactose into one molecule of glucose and one molecule of lactose to achieve the result of lactose cleavage.

Lactobacillus fermentans can produce $\alpha$-lactose, and its maximum enzyme activity is $21.38 \mathrm{u} / \mathrm{ml}$. Lactobacillus fermentans and Bifidobacterium longum can produce $\alpha$-lactose, and the enzyme activity can reach $22.41 \mathrm{u} / \mathrm{ml}$. Many studies have shown that Lactobacillus bulgaricus can produce $\beta$-lactose. Finally, a high-yield $\beta$-lactose strain was obtained from Yunnan Tempe samples, which was identified as Lactobacillus brevis [21]. In conclusion, probiotics can produce antioxidant-related enzymes during development and reproduction. Among them, there are many researches on SOD, and the activity of SOD produced by different preventive drugs is significantly different. In addition to producing enzymes related to antioxidants, probiotics may also produce other enzymes related to normal function [22].

2.4. Function of Feed Enzyme. The cell wall of wheat food is rich in protein, lipids, and polysaccharides without starch. These substances are linked to various chemical bonds, such as peptide bonds. The digestive enzymes in the digestive tract of monogastric animals cannot decompose polysaccharides without starch. Protein and starch in cells are released from cells by preparing nonstarch polysaccharide enzymes, which destroy the structure of the plant cell wall and participate in the normal metabolism of the body. Cellulose can significantly improve the digestibility of cell wall components, increase the dissolution rate of minerals, improve the production efficiency of chicken, and improve the utilization rate of nutrients [23].

Many metabolites in feed cannot be hydrolyzed by endogenous enzymes. These metabolites have antinutritional effects on animals and prevent the digestion and utilization of nutrients such as protein, fat, and starch. For feeds that have antinutritional effects on animals, such as nonstarch polysaccharides, phytic acid, phytosanitary hemolysin, and protease inhibitors [24], enzyme preparations can partially or completely eliminate the antinutritional effects of antinutrients and improve feed utilization. Adding the nonstarch polysaccharide enzyme and protein and planting enzyme preparation in the diet can significantly improve the utilization rate of nutrients and improve livestock and poultry production efficiency, reduce environmental pollution, and improve economic benefits [25].

\section{Experimental Design and Analysis}

3.1. Preparation of Materials. There were four groups of probiotics, including CG in the drinking water control group, KJT of Lactobacillus plantarum, DSSJ of Clostridium butyricum, and DB of Bacillus licheniformis. Lactating chickens come from a farm in Zhejiang Province, and their feed is provided by a vegetable market in Zhejiang Province.

Before the experiment, the chickens were starved for eight hours. When slaughtering, they need to be put on a board, their heads are cut off and killed, and then they are dissected quickly, the gastrointestinal tract is removed, and the whole digestive system separates the two parts of the intestine in the stomach with scissors. The tissue samples were cut with scissors, and part of the connective tissue was removed and washed with cold saline water to remove the remaining contents. Filter paper is used to absorb the water on the surface, and 5 times the volume of cold brine solution is weighed and added. After homogenization, it is used and centrifuged at the speed of $1200 \mathrm{R} / \mathrm{min}$ on the ultralow temperature high-speed freezing centrifuge. After the supernatant was taken, it was the original enzyme solution, which was frozen and stored at $30^{\circ} \mathrm{C}$. The frozen samples should be avoided to prevent from affecting the enzyme activity.

3.2. In Vitro Digestion Experiment. First, the weighing dish was dried in an oven at $530^{\circ} \mathrm{C}$ to the weight of the solid and it was weighed. About $2 \mathrm{~g}$ of the feed sample was weighed, it was put in the weighing dish and dried for 3 hours, it was transferred to the dryer to cool for half an hour, and it was weighed, it was put in the oven for 45 minutes, and it was moved to the dryer to cool for 20 minutes. Drying was repeated until the weight remains unchanged. The mass lost during drying is the weight of water. The dried feed sample was put into a $100 \mathrm{ml}$ conical flask, $10 \mathrm{ml}$ of homogeneous raw stomach enzyme solution was added, and then $10 \mathrm{ml}$ PBS adjustable solution was added. The $\mathrm{pH}$ was adjusted to about 3 with $\mathrm{HCI}$, and then $2 \mathrm{ml}$ of $60 \mathrm{u} / \mathrm{ml}$ penicillin was 
added, once every hour. After adding the rubber cover, the rubber cover was placed in a $25^{\circ} \mathrm{C}$ constant temperature shaker for 60 times/min and shaken for 2 hours. In the second stage, the conical flask from the shaking table was taken out, sodium hydroxide solution was added, the $\mathrm{pH}$ of the system was adjusted to about 6.5 , and then $20 \mathrm{ml}$ of intestinal homogenate enzyme solution, $15 \mathrm{ml}$ of PBS adjustable solution, and finally $2 \mathrm{ml}$ of $60 \mathrm{u} / \mathrm{ml}$ streptomycin were added, once every two hours. After the rubber cap was added, it was placed in a thermostatic stirrer at $25^{\circ} \mathrm{C}$ for 60 times/min for 8 hours. In the third stage, $1 \mathrm{ml}$ of $35 \%$ trichloroacetic acid was added to the cone, and the enzyme reaction was stopped for 20 minutes. Then, the residue was washed with ethanol and acetone, respectively, with quantitative filter paper of known quality, and the residue was collected and dried and weighed.

3.3. Test Index. Preparation of the reducing sugar curve: $3 \mathrm{ml}$ acetic acid and sodium acetate control solution were absorbed, $2 \mathrm{ml}$ ldns reagent was added and heated in boiling water for 8 minutes, cooled with tap water at room temperature and added $20 \mathrm{ml}$ distilled water to make a typical blank sample. Glucose standard solution with concentrations of $0.3-0.5 \mathrm{mg} / \mathrm{ml}$ was prepared by diluting glucose solutions of $1,2,3,4$, and $5 \mathrm{ml}$ pipettes to $50 \mathrm{ml}$ with sodium acetate and acetic acid, respectively. $100 \mathrm{ml}$ each of the above standard glucose solutions in the above concentration sequence (two parallel) into the calibration tube were put, and then $1 \mathrm{ml}$ acetic acid, sodium acetate, and $4 \mathrm{ml}$ ldns reagent were added. Electromagnetic oscillation for $3 S$ and heating in boiling water for 5 minutes were done. After that, the samples were cooled with tap water at room temperature; distilled water was added to $30 \mathrm{ml}$ volume, and the OD absorption value was determined at $380 \mathrm{~nm}$. The standard curve was drawn $(y=a x+b)$.

After enzymolysis, the sample was filtered into an $80 \mathrm{ml}$ volumetric flask under vacuum. $1 \mathrm{ml}$ of the solid volume filter was taken and $3 \mathrm{ml}$ of acetic acid was added. Sodium acetate adjustable solution and reagent $4 \mathrm{ml}$ LDNS is mixed and boiled in boiling water for 10 minutes. Then, it was diluted to $30 \mathrm{ml}$ with distilled water. After mixing, the color was compared with $380 \mathrm{~nm}$. The content of reducing sugar was calculated. The blank control group was added with $1 \mathrm{ml}$ water, $3 \mathrm{ml}$ acetic acid, and $4 \mathrm{ml}$ sodium acetate-regulating solution. The net yield of reducing sugar $=$ the amount of reducing sugar produced in the enzyme solution-the amount of reducing sugar produced in the blank solution. The compound enzyme after enzymatic hydrolysis of the feed sample is measured. Measurement method: the residue was filtered and digested under vacuum, and finally it was dried to constant weight with nitrogen-free filter paper of known weight in an oven at $100^{\circ} \mathrm{C}$, and then the energy value of the residue was weighed and measured.

3.4. Experimental Theory. When analyzing and studying the relationship between experimental data, the relationship between any two variables is called correlation. The correlation among test data includes linear correlation, exponential correlation, and arithmetic correlation. Among them, linear association is the simplest and the most studied one. Other different associations can also be transformed into linear association through specific transformation. The reliability of the results of this study is based on the statistical analysis method of correlation analysis. The experiments of four probiotics and a variety of enzymes on chickens were carried out, and the relationship between them was discussed. In this paper, Pearson correlation factor is calculated by linear correlation analysis. The Pearson correlation factor is used to measure whether all data points belong to a straight line, that is, the description of the correlation between two variables. The calculation formula is as follows:

$$
r=\frac{N \sum x_{i} y_{i}-\sum x_{i} y_{i}}{\sqrt{N \sum x_{i}^{2}-\left(\sum x_{i}^{2}\right)} \sqrt{N \sum y_{i}^{2}-\left(\sum y_{i}^{2}\right)}} .
$$

The Pearson correlation coefficient is between -1 and 1 . When the correlation coefficient approaches -1 to 1 , the stronger the correlation is, the weaker the correlation will be. Results belonging to $0.8-1$ means extremely strong correlation; results belonging to $0.6-0.8$ indicate strong correlation; results belonging to $0.4-0.6$ indicate moderate correlation; results belonging to $0.2-0.4$ indicate weak correlation; and results belonging to $0-0.2$ indicate extremely weak correlation or no correlation.

\section{Influence of Probiotics and Compound Enzyme}

4.1. Test Results of Chicken Growth Performance. Four experimental groups were set up: control group CG with drinking water, KJT with Lactobacillus plantarum, DSSJ with Clostridium butyricum, and DB with Bacillus licheniformis, and each experimental group was tested three times to ensure the accuracy of the experiment. The experimental data are shown in Table 1.

For these experimental objects, the weight of these chickens was detected at 5 days, 10 days, and 15 days respectively, and the weight of these chickens in the corresponding time period was recorded, so as to analyze the influence of different weights produced by different probiotics, as shown in Figure 1.

In the figure, we can clearly see the weight change of the chicken. In these 15 days, the weight gain of the experimental group of Clostridium butyricum was the most obvious, and the weight was as high as $1.16 \mathrm{Jin}$, and in 5 days, 10 days, and 15 days of these three periods are the heaviest weight, with the average daily gain of 0.39 Jin. The results of the Lactobacillus plantarum experimental group and Clostridium butyricum experimental group had no significant difference, which were within 0.1 Jin, and had great effect on weight, with an average weight gain of 0.3 Jin. The weight gains of the control group with only drinking water were $1.38,1.47$, and 1.63 Jin, respectively, which were the least weight gain group, and the difference between the two groups was significant. The weight gains of Bacillus licheniformis experimental group were 1.5, 1.81, and $2.42 \mathrm{Jin}$, 
TABLE 1: Test results of growth performance.

\begin{tabular}{lccc}
\hline Experiment & Group & Repeat & Treating \\
\hline 1 & CG & 3 & Drinking water \\
2 & KJT & 3 & Drinking water + 0.3\% Lactobacillus plantarum \\
3 & DSS & 3 & Drinking water $+0.3 \%$ Clostridium butyricum \\
4 & DB & 3 & Drinking water + 0.3\% Bacillus licheniformis \\
\hline
\end{tabular}

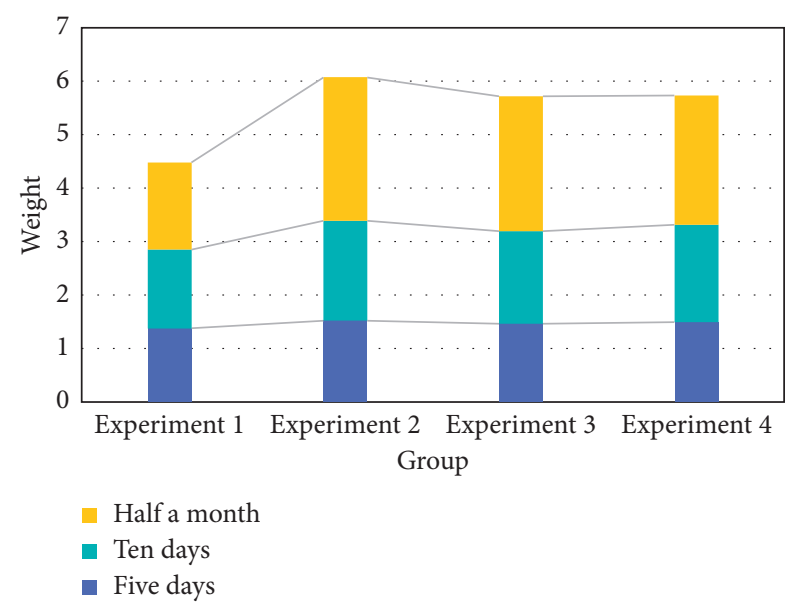

FIgURE 1: Weight change pattern.

respectively. Although the effect of probiotics was not obvious in the other two groups, it still had great advantages compared with the control group.

\subsection{Apparent Digestibility of Compound Enzyme Preparation} in the Terminal Ileum and Total Digestive Tract (\%). The compound enzyme, which is mainly composed of cellulose and pectinase, may destroy the plant cell wall, release nutrients in the cells, easily contact with digestive enzymes, and eliminate antinutrients in diet, so as to reduce food viscosity and gastrointestinal contents and promote animal digestion and absorption. If the rate of gastric emptying slows down and the amount of digestive juice discharged per unit time decreases, the digestion of nutrients is limited, as shown in Figure 2.

As can be seen from the figure, most of the available nutrients in the food have been released before they enter the animal body. On the other hand, the decrease of the geometric mean size of food may also increase the intestinal digestibility. In four groups of experiments, the ileum terminal, dry matter, crude protein, and organic compounds were detected. Under the condition of drinking water, the digestibility of these four substances were $67.8 \%, 72.3 \%$, $54.1 \%$, and $70.4 \%$, respectively. In the third group, the digestibility was the highest, $78.3 \%, 80.2 \%, 63.8 \%$, and $79.1 \%$, respectively. The excellent digestive system also led to the heaviest weight of this chicken. Secondly, the digestive capacity of the second group was catalyzed by Lactobacillus plantarum, and the last group was Bacillus licheniformis. From the data in the figure, we can not only see the influence of the four probiotics on the digestibility but also find the digestibility performance of the four probiotics in the terminal ileum and the whole digestive tract. The digestibility of

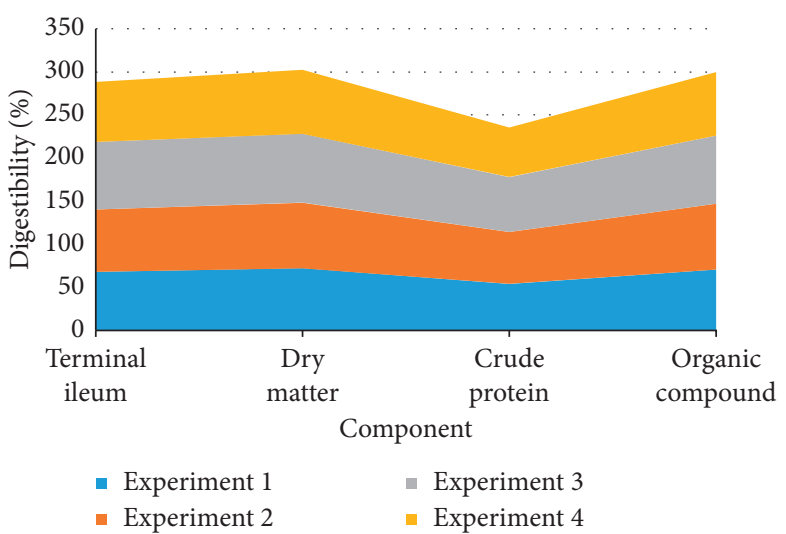

Figure 2: Apparent digestibility.

the dry matter and organic compounds is the best, with the digestibility of above $70 \%$, while the crude protein is the worst, with the digestibility of about $50 \%-60 \%$.

4.3. Effect of $\beta$-Amylase on the Digestive Tract. After saccharification experiment with $\beta$-amylase, the content of reducing sugar was measured every 1 hour, the relationship between the reduction of sugar in the intestinal tract and the change of time and the relationship between the content of reducing sugar and the amount of $\beta$-amylase, as shown in Figure 3.

With the passage of time, the content of reducing sugar in the intestinal tract increased significantly in the process of $1-3$ hours, indicating that $\beta$-amylase has a certain catalytic effect on the degradation of starch in the process of reducing sugar, and the content of reducing sugar slightly increased on both sides of 3-4 h, but not obviously. This may be related to the total amount of residues in the intestine. With the increase of saccharification time, the content of reducing sugar increased slightly, indicating that saccharification was basically mature. Assuming that the amount of $\beta$-amylase increases, the sugar rate of the sample will increase, thus increasing the content of reducing sugar in the solution. In the initial stage of the saccharification process, the $\beta$-amylase must maintain sufficient activity under certain conditions, while the initial stage of saccharification cannot provide proper temperature and $\mathrm{pH}$ environment for $\beta$-amylase two hours before the beginning of saccharification, so the content of reducing sugar in the reaction solution has little change. As the reaction time goes on, when the reaction time is long enough to 3 hours, the reducing sugar content in the reaction solution will increase rapidly, especially, when the enzyme concentration in the reaction solution increases, the rate at which the sugar content decreases also increases. 


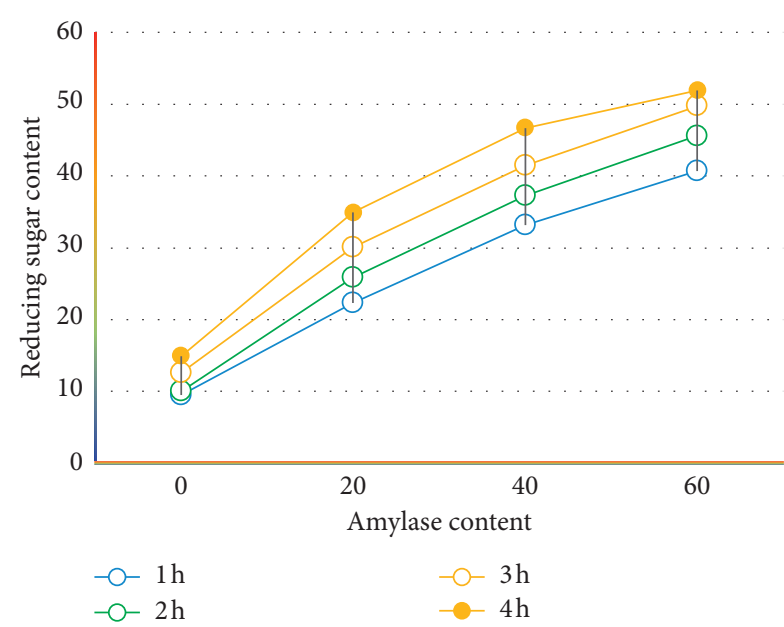

Figure 3: Change of reducing sugar content.

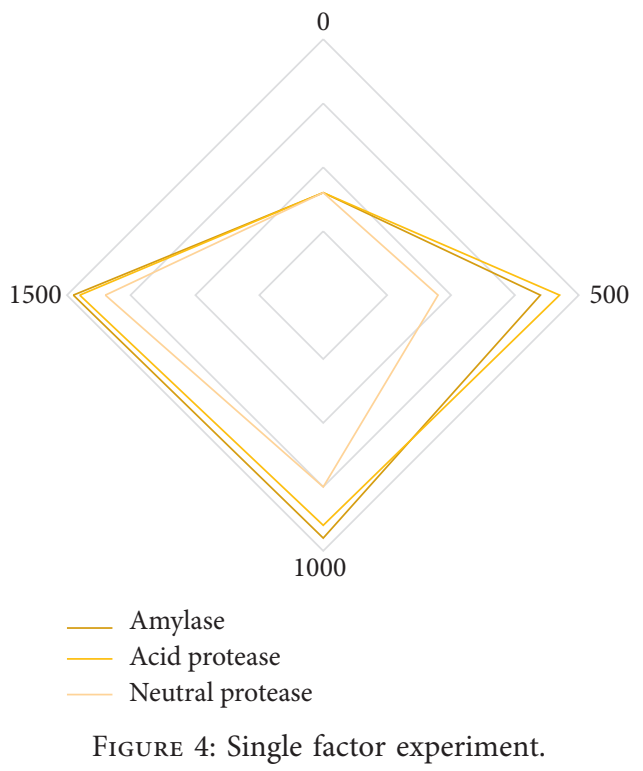

When the concentration of $\beta$-amylase in the reaction solution is $30 \mathrm{U} / \mathrm{g}$, the yield of reducing sugar reaches the maximum value, so the optimal addition amount of $\beta$-amylase is $30-40 \mathrm{u} / \mathrm{g}$.

4.4. Single Factor Experiment. In the single factor experiment, amylase, acid protease, and neutral protease should be added into the conical flask, respectively, and the enzyme contents are 500, 1000, and $1500(\mathrm{U} / \mathrm{g})$. Then, the experimental results obtained from the three groups of data are detected, respectively. The in vitro digestibility is affected by different enzyme types and different contents, as shown in Figure 4.

From the data in the figure, it can be seen that the feed digestibility of the enzyme group is higher than that of the control group. The general trend of adding enzyme preparation is rapidly increased and then tend to be gentle, but on the whole, it is gradually increasing. The digestibility of amylase increased from $16 \%$ to $39 \%$ with the increase of the enzyme content. The digestibility of acid protease increased from $16 \%$ to $38 \%$. The digestibility of neutral protease at $1500(\mathrm{U} / \mathrm{g})$ was 34\%. If the enzyme was added later, the digestibility would increase. The enzyme activity will be seriously affected by temperature, too low or too high will make the enzyme lose activity; when the temperature is most appropriate, the enzyme activity is the highest, the impact on digestibility is the best, and the ability to promote digestion is the strongest.

\section{Conclusions}

The intestinal tract of chicks is aseptic at birth. After birth, with the environmental contact of lactation and delivery clinic, foreign bacteria gradually enter into the intestine. After attachment and colonization, the intestinal flora is created to maintain its own intestinal microecological balance. Therefore, Lactobacillus and other beneficial bacteria are used to complete infant breast-feeding so that the beneficial bacteria occupy the effective position of the intestinal tract earlier, which has an absolute advantage in quantity and action intensity, effectively inhibit the attachment of other pathogenic bacteria to the site, and effectively prevent the generation and invasion of harmful bacteria. Compound probiotics is an important antibacterial compound. Lactic acid production can reduce the intestinal $\mathrm{pH}$ value and inhibit the growth of pathogenic bacteria such as Escherichia coli and Salmonella. By consuming oxygen, Bacillus creates an environment conducive to the growth of anaerobes (such as lactic acid bacteria and Bifidobacteria) or anaerobic bacteria and prevents the reproduction of Escherichia coli and Salmonella, and maintains the balance of intestinal flora to prevent the occurrence of diseases. The bacteria and lactic acid bacteria in the compound preparation can produce rich digestive enzymes, organic acids, and vitamins.

Enzyme preparation is a kind of safe, reliable, and pollution-free green feed additive. It has the ability of biocatalysis reaction, which can improve the production efficiency of livestock and poultry, reduce environmental pollution, and save animal feed resources. Enzyme preparation can improve the utilization rate of feed and raw materials by decomposing the antinutritional agents in feed and affect the digestion and metabolism of animals. The flora found in the digestive tract is considered a new nutrient because it is closely related to the health of host diseases, metabolism, and regulation of the immune system. They allow the host to increase the absorption of nutrients and affect energy intake. Studies have shown that there are many factors affecting the distribution and stability of intestinal microflora, such as the changes of environment, seasons, and the role of food additives, so that the intestinal microorganisms affect the development of animals to a certain extent. As a kind of food additive, probiotics play a beneficial role in the intestinal flora. The addition of probiotics can help the host control harmful bacteria and have beneficial effects on intestinal flora. In addition, probiotics can also regulate the immune system to fight against various diseases to produce resistance to diseases. 
The evaluation methods of the enzyme nutritional value of compound feed mainly include the in vivo method and in vitro method. The in vivo method is an animal feeding test and metabolism test, also known as the biological method. This is a logical and scientific test method, but it costs a lot of manpower and time. It is seriously affected by external factors (climate, environment, human function, etc.) and it is not easy to evaluate a large amount of feed or food in a short time. The in vitro method was used to simulate the digestive system of monogastric animals to evaluate the nutritional value of food. The method is rapid, simple, laborsaving, and time-saving. In this experiment, the effects of probiotics and complex enzymes on the chicken digestive tract were studied by using this technology. The net yield of reducing sugar and the total energy of the digestive tract residue were taken as indexes. The results showed that the compound enzyme preparation and probiotics played an important role in the growth and development of the chicken.

\section{Data Availability}

No data were used to support this study.

\section{Conflicts of Interest}

The authors declare no conflicts of interest.

\section{Authors' Contributions}

Ruokun Yi performed the majority of the experiments and wrote the manuscript. Yanni Pan and Xingyao Long contributed to the data analysis. Xin Zhao and Fang Tan designed and supervised the study and checked the final manuscript.

\section{Acknowledgments}

This research was funded by the Children's Research Institute of National Center for Schooling Development Programme and Chongqing University of Education (CSDP19FS01103), the Science and Technology Research Program of Chongqing Municipal Education Commission (KJZD-K201901601), and Research Project of Chongqing University of Education (KY2015TBZC), China.

\section{References}

[1] J. Li, N. Sung, Y. Ni, G. Panagiotou, and H. El-Nezami, "Probiotics modulated gut microbiota suppresses hepatocellular carcinoma growth in mice," Proceedings of the $\mathrm{Na}$ tional Academy of Sciences, vol. 113, no. 9, pp. E1306-E1315, 2016.

[2] R. Tanaka, M. Aoki, T. Miyazaki, H. Mitsuya, M. Ootsubo, and P. Bossier, "In situ enumeration and localization of the probiotic Pediococcus sp. strain Abl in the gut of abalone Haliotis gigantea," Fisheries Science, vol. 82, no. 3, pp. 481-489, 2016.

[3] K. Balasingham, L. Radhakrishnan, C. Valli, and D. Balasuramanyam, "Probiotic characterization of lactic acid bacteria isolated from swine intestine," Veterinary World, vol. 10, no. 7, pp. 825-829, 2017.

[4] L. Jacobs, "Probiotics, prebiotics, and synbiotics," Journal of Pediatric Surgical Nursing, vol. 6, no. 3, pp. 53-55, 2017.

[5] R. R. Watson and V. R. Preedy, "Probiotics, prebiotics, and synbiotics," Journal of Food Ence \& Technology, vol. 52, no. 12, pp. 7577-7587, 2015.

[6] M. Panduru, N. M. Panduru, C. M. Sălăvăstru, and G.-S. Tiplica, "Probiotics and primary prevention of atopic dermatitis: a meta-analysis of randomized controlled studies," Journal of the European Academy of Dermatology and Venereology, vol. 29, no. 2, pp. 232-242, 2015.

[7] C. A. Cuello-Garcia, J. L. Brożek, A. R. Fiocchi et al., "Probiotics for the prevention of allergy: a systematic review and meta-analysis of randomized controlled trials," Journal of Allergy and Clinical Immunology, vol. 136, no. 4, pp. 952-961, 2015.

[8] K. Chau, S. E. Lau, S. Greenberg, P. Yazdani-Brojeni, S. Jacobson, and G. Koren, "Probiotics for infantile colic: a randomized, double-blind, placebo-controlled trial investigating lactobacillus reuteri DSM 17938," The Journal of Pediatrics, vol. 166, no. 1, pp. 74-78, 2015.

[9] P. D. Cani and M. Van Hul, "Novel opportunities for nextgeneration probiotics targeting metabolic syndrome," Current Opinion in Biotechnology, vol. 32, no. 32, pp. 21-27, 2015.

[10] C. Hicks, S. Moore, and A. Andrade, "Probiotics for the prevention of antibiotic-associated diarrhea," Explore the Journal of Ence \& Healing, vol. 12, no. 6, pp. 463-466, 2016.

[11] P. Markowiak and K. Śliżewska, "Effects of probiotics, prebiotics, and synbiotics on human health," Nutrients, vol. 9, no. 9, pp. 1-12, 2017.

[12] P. A. Bron, M. Kleerebezem, P. D. Cani et al., "Can probiotics modulate human disease by impacting intestinal barrier function?" British Journal of Nutrition, vol. 117, no. 1, pp. 93-107, 2017.

[13] S. Cruchet, A. R. Furnes, J. Palacios, E. Hebel et al., "The use of probiotics in pediatric gastroenterology: a review of the literature and recommendations by Latin-American experts," Pediatric Drugs, vol. 17, no. 3, pp. 199-216, 2015.

[14] J. Plaza-Díaz, F. Ruiz-Ojeda, L. Vilchez-Padial, and A. Gil, "Evidence of the anti-inflammatory effects of probiotics and synbiotics in intestinal chronic diseases," Nutrients, vol. 9, no. 6, p. 555, 2017.

[15] M. Sáez-Lara, C. Robles-Sanchez, F. Ruiz-Ojeda, J. Plaza-Diaz, and A. Gil, "Effects of probiotics and synbiotics on obesity, insulin resistance syndrome, type 2 diabetes and non-alcoholic fatty liver disease: a review of human clinical trials," International Journal of Molecular Sciences, vol. 17, no. 6, p. 928, 2016.

[16] S. Park and J.-H. Bae, "Probiotics for weight loss: a systematic review and meta-analysis," Nutrition Research, vol. 35, no. 7, pp. 566-575, 2015.

[17] A. G. Cid, M. C. Goldner, M. Daz, and G. Ellenrieder, "The effect of endozym $\beta$-split, a commercial enzyme preparation used for aroma release, on tannat wine glycosides[J]," South African Journal for Enology \& Viticulture, vol. 33, no. 1, pp. 51-57, 2016.

[18] L. T. Piemolini-Barreto, R. V. Antônio, and S. Echeverrigaray, "Comparison of a pectinolytic extract of Kluyveromyces marxianus and a commercial enzyme preparation in the production of Ives (Vitis labrusca) grape juice," World Journal of Microbiology and Biotechnology, vol. 31, no. 5, pp. 755-762, 2015. 
[19] K. R. Kishore, "Targeting glucocerebrosidase: reduced enzymatic activity and Parkinson's disease," Movement Disorders, vol. 30, no. 12, p. 1620, 2015 .

[20] K. Ziervogel, S. B. Joye, and C. Arnosti, "Microbial enzymatic activity and secondary production in sediments affected by the sedimentation pulse following the Deepwater Horizon oil spill," Deep Sea Research Part II: Topical Studies in Oceanography, vol. 129, pp. 241-248, 2016.

[21] M. S. Islam and D. J. Moore, "Mechanisms of LRRK2-dependent neurodegeneration: role of enzymatic activity and protein aggregation," Biochemical Society Transactions, vol. 45, no. 1, pp. 163-172, 2017.

[22] J. F. Siebel, A. Adamska-Venkatesh, S. Rumpel, and K. Weber, E. Reijerse and W. Lubitz, Hybrid [FeFe]-Hydrogenases with modified active sites show remarkable residual enzymatic activity," Biochemistry, vol. 54, no. 7, pp. 1474-1483, 2015.

[23] A. Piotrowska-Długosz and P. Charzynski, "The impact of the soil sealing degree on microbial biomass, enzymatic activity, and physicochemical properties in the Ekranic Technosols of Torun (Poland)," Journal of Soils \& Sediments, vol. 15, no. 1, pp. 47-59, 2015.

[24] D. Zou, Y. Huang, X. Zhao et al., "A novel New Delhi metallo$\beta$-lactamase variant, NDM-14, isolated in a Chinese hospital possesses increased enzymatic activity against carbapenems," Antimicrobial Agents and Chemotherapy, vol. 59, no. 4, pp. 2450-2453, 2015.

[25] F. I. Ndwiga, "Enzymatic activity, and secondary metabolites of fungal isolates from lake sonachi in Kenya," International Journal of Imaging Systems \& Technology, vol. 21, no. 2, pp. 211-222, 2017. 